Review Article

\title{
Effects of Health Literacy Intervention on Health Literacy Level and Glucolipid Metabolism of Diabetic Patients in Mainland China: A Systematic Review and Meta-Analysis
}

\author{
Yangli Chen $(\mathbb{D}$, Xue Ran $\mathbb{D}$, Yalan Chen $\mathbb{D}$, and Kui Jiang $\mathbb{D}$ \\ Department of Medical Informatics, Medical School of Nantong University, Nantong 226001, China \\ Correspondence should be addressed to Yalan Chen; ylchen@ntu.edu.cn and Kui Jiang; kuij@ntu.edu.cn
}

Received 21 July 2021; Revised 6 November 2021; Accepted 9 December 2021; Published 30 December 2021

Academic Editor: Akira Sugawara

Copyright (c) 2021 Yangli Chen et al. This is an open access article distributed under the Creative Commons Attribution License, which permits unrestricted use, distribution, and reproduction in any medium, provided the original work is properly cited.

\begin{abstract}
Objective. To systematically evaluate the effects of health literacy intervention on health literacy level and glycolipid metabolism of people with diabetes in mainland China. Methods. A systematic review of journal articles discussing diabetes and health literacy was performed by searching PubMed, Embase, the Science Citation Index Expanded (SCIE) database of Web of Science, the China National Knowledge Infrastructure (CNKI) database, the Chinese Scientific and Technical Journals database (CQVIP), and the Wanfang database. Cochrane Effective Practice and Organization of Care Review Group (EPOC) standards were applied for quality assessment. A meta-analysis was performed using Stata 12.0 software. Results. A total of 44 articles, including seven controlled before-and-after trials (CBAs), 27 randomized controlled trials (RCTs), and 10 nonrandomized controlled trials (non-RCTs), were included. The results showed that (1) health literacy level in the intervention group was improved compared with the preintervention and the control group; (2) fasting plasma glucose (FPG) (standardized mean difference $(\mathrm{SMD})=-1.85$, 95\% CI: $-2.28,-1.42)$, 2-hour plasma glucose (2hPG) ( $\mathrm{SMD}=-2.18,95 \% \mathrm{CI}:-2.68,-1.68$ ), and HbA1c (weighted mean difference $(\mathrm{WMD})=-1.21,95 \% \mathrm{CI}:-1.48,-0.94)$ were significantly reduced in the intervention group; (3) total cholesterol (TC) $(\mathrm{WMD}=-0.43,95 \% \mathrm{CI}:-0.64,-0.23)$ was significantly reduced in the intervention group, although there were no statistically significant differences for triglycerides (TG) (WMD $=-0.34,95 \% \mathrm{CI}:-0.73,0.05)$, low-density lipoprotein cholesterol (LDL-C) $(\mathrm{WMD}=-0.20,95 \% \mathrm{CI}:-0.46,0.07)$, or high-density lipoprotein cholesterol (HDL-C) (WMD $=-0.06,95 \% \mathrm{CI}:-0.29,0.17)$. Conclusion. Intervention based on health literacy can effectively improve health literacy levels and reduce glucose metabolism and TC level among people with diabetes mellitus, although it has no significant effect on TG, LDL-C, or HDL-C.
\end{abstract}

\section{Introduction}

According to the latest global diabetes map released by the International Diabetes Federation (IDF), approximately 463 million adults worldwide were diagnosed with diabetes in 2019. China had the largest population of people with diabetes, at approximately 116.4 million people [1]. Diabetes has become a major chronic disease impacting human health and bearing a considerable health and economic burden on society.

Diabetes health education (HE) has always been recognized as the cornerstone of effective diabetes management [2], while health literacy (HL) is an important component of diabetes $\mathrm{HE}$ and health promotion theories. Diabetes HL refers to an individual's ability to acquire, process, and understand diabetes-related information and medical services [3], which include the ability to read, comprehend, calculate, and utilize basic diabetes-related health information [4-6].

Interventions for people with diabetes with low HL have been implemented in Europe and America. After introducing HL intervention in 250 patients with type 2 diabetes, Kim et al. demonstrated outstanding reductions in glycated hemoglobin $(\mathrm{HbA} 1 \mathrm{c})$ and statistically significant improvement in patient-reported measures of diabetes burden and quality of life in the intervention group [7]. Chamany et al. 
found that $\mathrm{HbAlc}$ was significantly lower in the intervention group than the control group after implementation of diabetes self-management and telephone intervention. Both groups experienced similar improvements in self-care activities, medication adherence, and intensification [8]. This research area has attracted enduring interest in mainland China, and several studies have explored the effects of HL interventions, although the results are inconsistent. For instance, Ouyang and Liang found that levels of HbAlc and fasting plasma glucose (FPG) were significantly reduced after personalized HE intervention, while the difference in blood lipid level was not statistically significant [9]. In contrast, research from $\mathrm{Xu}$ et al. showed that a community-hospital-family model of intervention could effectively control the blood sugar and blood lipids of people with diabetes and improve their HL [10].

Therefore, we conducted a systematic review (SR) to analyze published studies on HL intervention for people with diabetes in mainland China in order to summarize the effects on HL level and the glucolipid metabolism of patients. This research is expected to suggest new approaches for the implementation of diabetes intervention trials. From a broader perspective, our study could be used for reference in carrying out diabetes HL interventions in other countries.

\section{Materials and Methods}

2.1. Research Design. This study was conducted according to Preferred Reporting Items for Systematic Reviews and MetaAnalyses (PRISMA) 2020 statement [11] (Table S1).

2.2. Data Sources and Searches. PubMed, Embase, the Science Citation Index Expanded (SCIE) database of Web of Science, the China National Knowledge Infrastructure (CNKI) database, the Chinese Scientific and Technical Journals database (CQVIP), and the Wanfang database were used to search for journal articles about diabetes and HL published in English or Chinese from 2010 to 2021. The following search terms in Chinese and English were used: (diabetes mellitus OR dm OR diabetes OR diabetic mellitus OR diabetic OR mellitus) AND health literacy. We updated the search on March 30, 2021. The detailed search strategy for each database is shown in Table S2.

2.3. Study Selection. Two investigators assessed the potential studies independently according to predefined inclusion and exclusion criteria. Conflicting decisions were addressed by negotiation or through the further judgment of a third investigator.

Inclusion criteria were as follows: (1) intervention study; (2) subjects were people with diabetes in mainland China; (3) the intervention group received HL intervention, while the control group received routine care (or the study had no control); and (4) blood glucose and lipid levels were examined and reported, as well as the HL score.

Exclusion criteria were as follows: (1) subjects had serious complications such as diabetic nephropathy, (2) not an intervention study, (3) study outcome did not include data analysis or complete data, and (4) duplicate publications or similar studies published by the same research group.

2.4. Data Extraction and Quality Assessment. The following data were extracted from selected studies: (1) study characteristics (e.g., author, year of publication, and intervention procedures); (2) measured blood glucose and blood lipid levels (e.g., FPG, 2-hour plasma glucose (2hPG), and total cholesterol (TC)); and (3) information on the HL scale (e.g., domains and scores).

Cochrane Effective Practice and Organization of Care Review Group (EPOC) standards [12] were applied for quality assessment including (1) random sequence generation, (2) allocation concealment, (3) baseline measurement criteria, (4) baseline characteristics before intervention, (5) data comprehensiveness, (6) blind implementation of outcome measurement, (7) protection against contamination, (8) selective reporting, and (9) other risks of bias. "Yes" (lowbias risk), "Unclear," or "No" (high-bias risk) was assigned according to these nine considerations. The Grading of Recommendations Assessment, Development, and Evaluation (GRADE) approach was used to assess the quality of evidence for each outcome indicator [13].

2.5. Data Synthesis and Analysis. A meta-analysis was performed by using Stata (version 12.0). Heterogeneity among the included studies was evaluated using the $Q$ test and quantified using $I^{2}$. Studies were considered to be homogeneous if $I^{2}<50 \%$ and $P>0.1$, and then, a fixed effect model was used to calculate the effect size. In contrast, if studies were considered to be heterogeneous, a random effect model was used to estimate the effect size. For continuous variables, the weighted mean difference (WMD) and the standardized mean difference (SMD) were applied. For all analyses, a twotailed $P$ value $<0.05$ indicated statistical significance. A sensitivity analysis was performed to validate the stability of the outcomes, and funnel plots were used to identify potential publication bias.

\section{Results}

3.1. Search Results and Study Characteristics. A total of 3358 related studies were retrieved from the six databases, and 1938 remained after eliminating duplicates; 1883 papers were excluded following a review of the titles and abstracts of the papers. Ultimately, 44 papers [9, 10, 14-55] were eligible for inclusion, of which 41 were in Chinese [9, 10, 14-23, 25-33, 35-44, 46-55] and three were in English $[24,34,45]$. The flow of the selection process followed PRISMA guidelines and is shown in Figure 1.

Details of all included studies and the characteristics of the HL interventions, such as intervention methods and indicators, are summarized in Table 1.

3.2. Quality Assessment. Because it is difficult to apply a double-blind method for HL interventions and because the outcome indicators were objective endpoints, it was assumed that a single-blind evaluation was adopted in all studies. It is clearly stated in EPCO standards that allocation concealment for controlled before-and-after trial (CBA) studies 


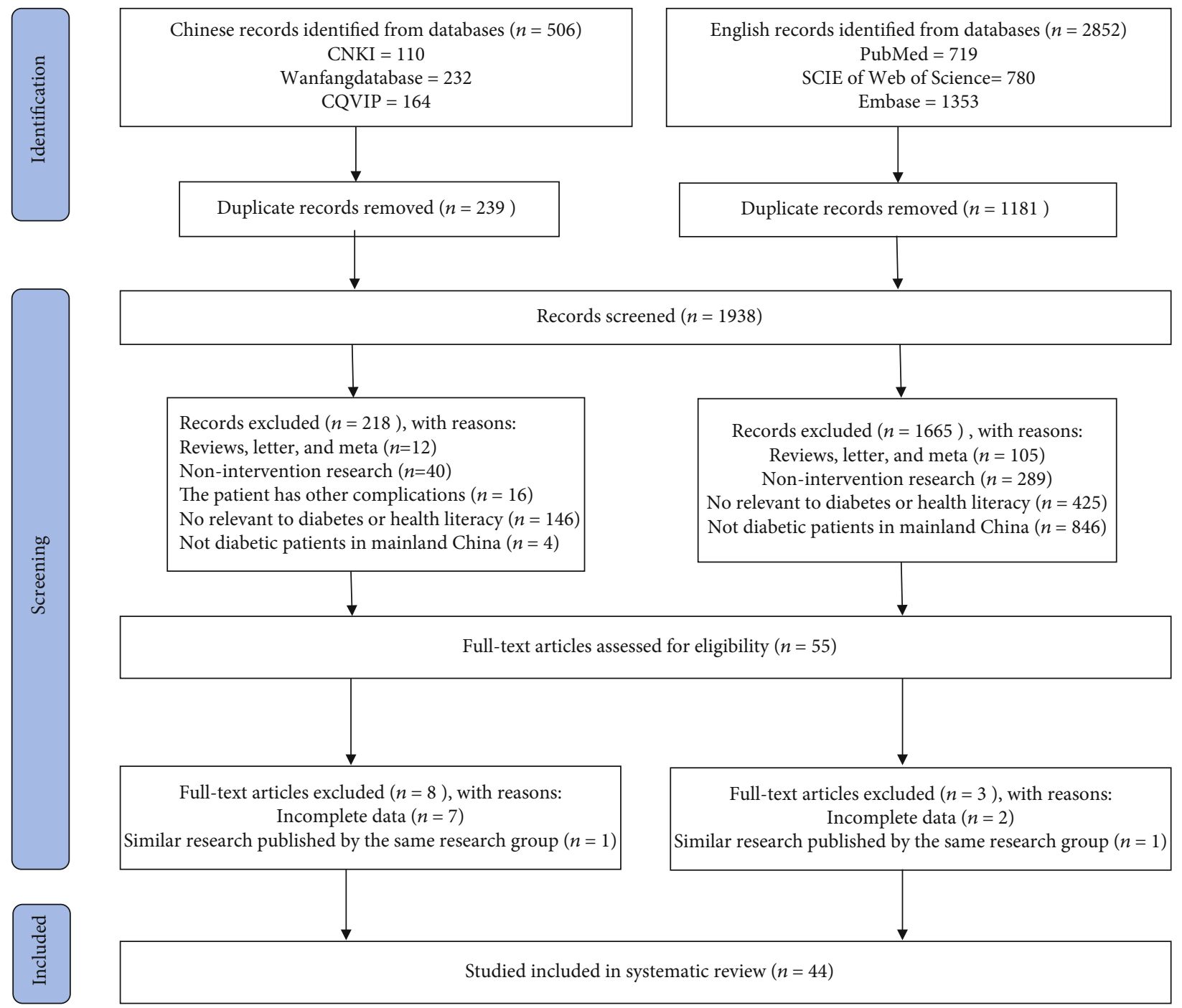

Figure 1: Article selection process.

should be scored "high risk" [12]. Among the included randomized controlled trial (RCT) studies, $22.2 \%$ did not specify the randomization method. None of the included studies described whether steps were taken to prevent data contamination. Overall, lack of random sequence generation, allocation concealment, and protection against contamination were the main sources of bias. A quality assessment of the included studies found low-bias risk scores over the nine areas ranging from 5 to 8 , indicating a medium overall quality evaluation (Table 2).

3.3. Overall Effect of Interventions on HL. A total of 31 studies $[9,10,14,15,17,19-21,25-29,31-33,35-38,41-44$, $46-50,52,55]$ used different HL assessment tools to analyze and report the impact of HL interventions on patient HL levels. As shown in Figure S1, self-designed questionnaires were utilized by about half of the studies $[14,17,20,21$, $26,28,35,37,38,41,46,48,49,52,55]$ and the Diabetes Health Literacy Assessment Tool designed by Miyong Kim was employed by one-quarter of the studies $[9,10,27,32$,
43, 44, 47]. Improving HL scores was regarded as an effective indicator by all the scales. Detailed evaluation of the contents of all assessment tools is shown in Table S3.

In terms of differences between the intervention and control groups after HL intervention, 20 studies [9, 10, 14, $15,17,19-21,25-27,29,31-33,35,36,38,41,52]$ showed that the total HL score of the intervention group was higher than that of the control group $(P<0.05)$, while two studies $[28,37]$ reported that the acceptability of HL of the intervention group was significantly better than that of the control group $(P<0.05)$.

In terms of pre- and postintervention changes in the intervention group, one study [43] found that there were significant differences in reading and comprehension skills $(P<0.05)$ before and after the intervention, except for numeracy skills $(P>0.05)$. Another study [50] indicated that after intervention, the proportion of patients with $\mathrm{HL}$ was higher than before $(P<0.05)$, while three studies [42, 48, 49] suggested that the HL level of people with diabetes was notably increased compared with preintervention. Finally, 


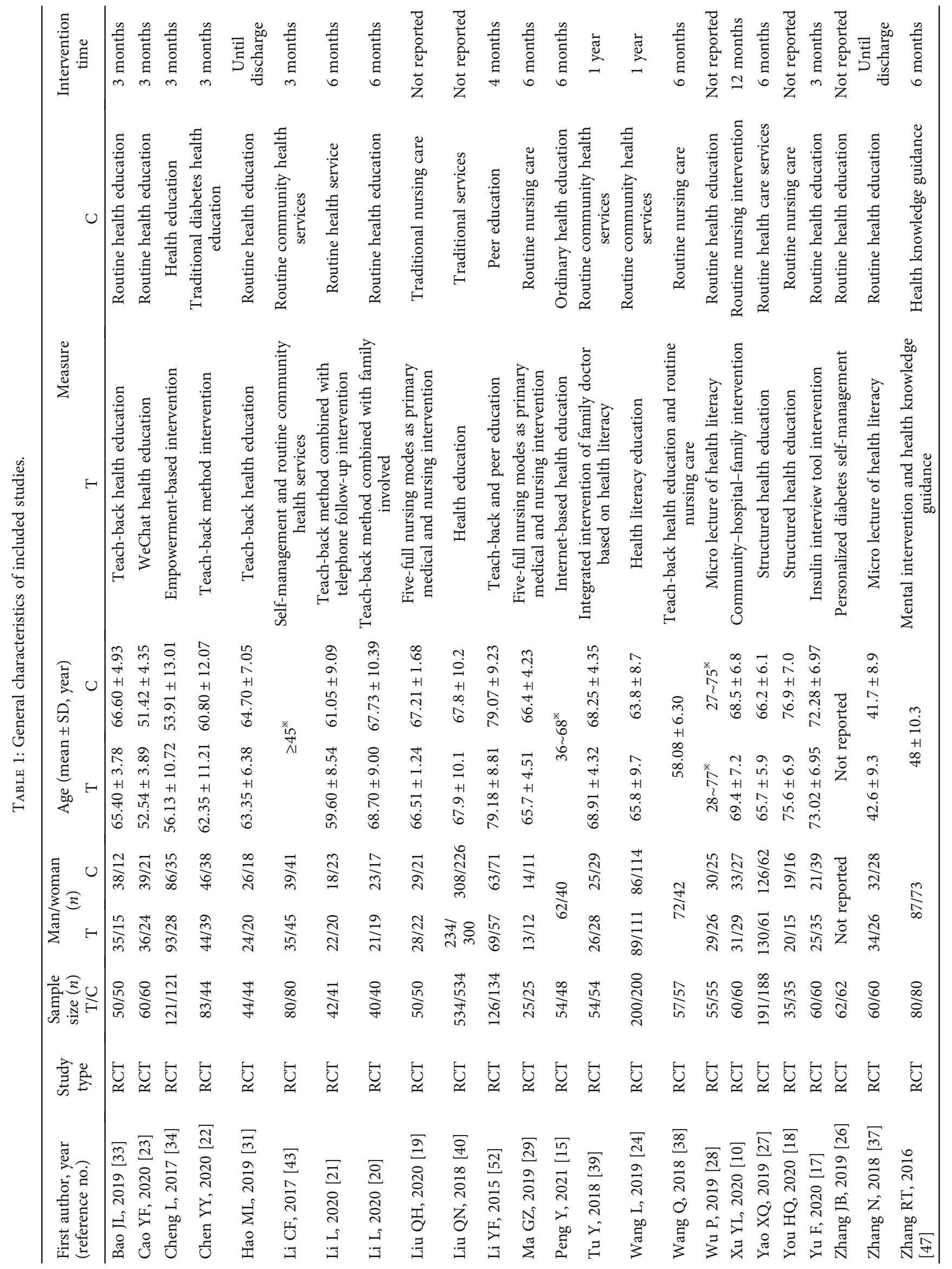




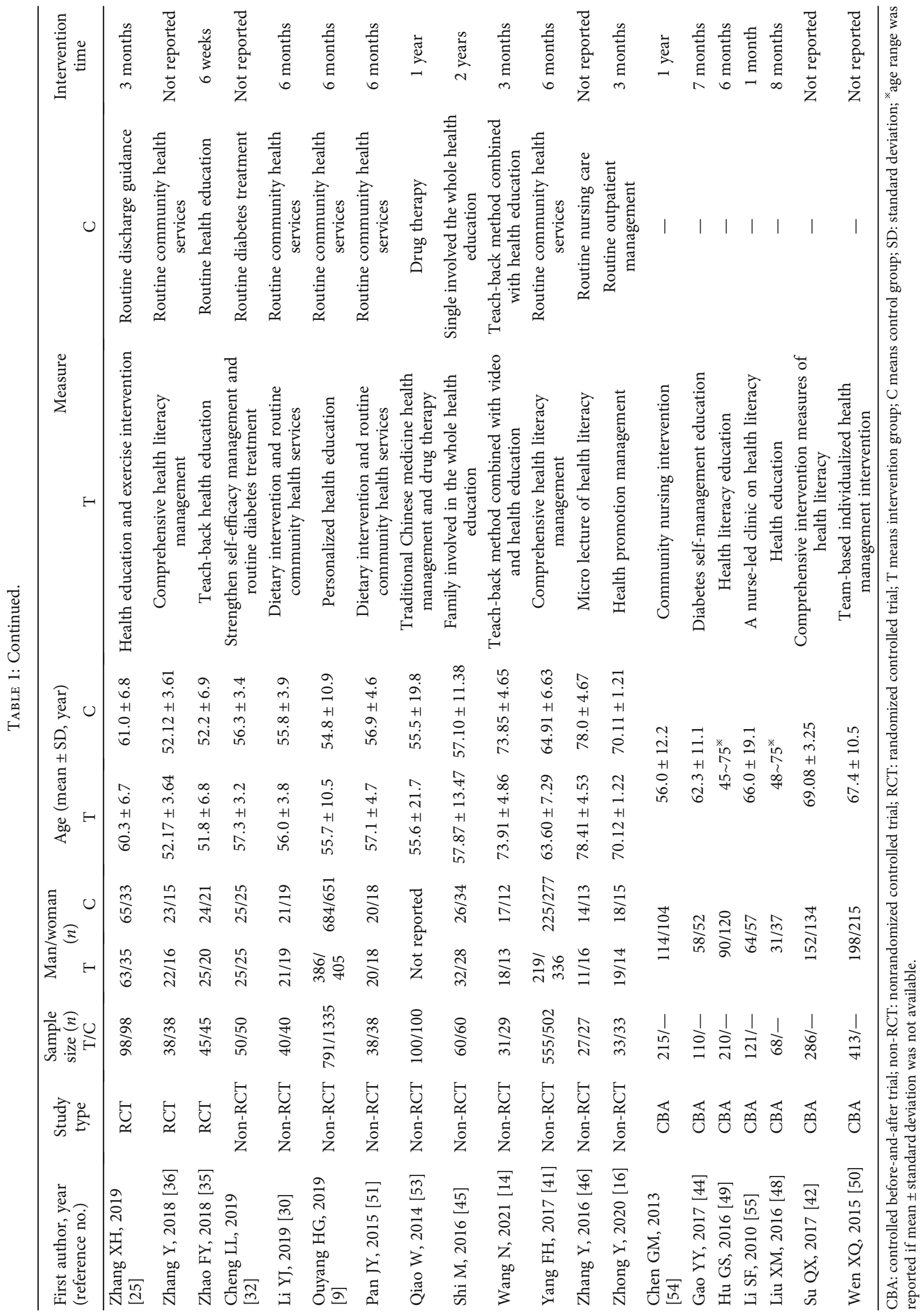


TABLE 2: Quality assessment of included studies.

\begin{tabular}{|c|c|c|c|c|c|c|c|c|c|c|c|}
\hline First author, year (reference no.) & Type & (1) & (2) & (3) & (4) & (5) & (6) & (7) & (8) & (9) & Score \\
\hline Bao JL, 2019 [33] & RCT & Yes & Unclear & Yes & Yes & Yes & Yes & Unclear & Yes & Yes & 7 \\
\hline Cao YF, 2020 [23] & RCT & Yes & Unclear & Yes & Yes & Yes & Yes & Unclear & Yes & Yes & 7 \\
\hline Cheng L, 2017 [34] & $\mathrm{RCT}$ & Yes & Yes & Yes & Yes & Yes & Yes & Unclear & Yes & Yes & 8 \\
\hline Chen YY, 2020 [22] & $\mathrm{RCT}$ & Yes & Unclear & Yes & Yes & Yes & Yes & Unclear & Yes & Yes & 7 \\
\hline Hao ML, 2019 [31] & $\mathrm{RCT}$ & Yes & Unclear & Yes & Yes & Yes & Yes & Unclear & Yes & Yes & 7 \\
\hline Li CF, 2017 [43] & $\mathrm{RCT}$ & Unclear & Unclear & Yes & Yes & Yes & Yes & Unclear & Yes & Yes & 6 \\
\hline Li L, 2020 [21] & $\mathrm{RCT}$ & Yes & Unclear & Yes & Yes & Yes & Yes & Unclear & Yes & Yes & 7 \\
\hline Li L, 2020 [20] & $\mathrm{RCT}$ & Yes & Unclear & Yes & Yes & Yes & Yes & Unclear & Yes & Yes & 7 \\
\hline Liu QH, 2020 [19] & RCT & No & Unclear & Yes & Yes & Yes & Yes & Unclear & Yes & Yes & 6 \\
\hline Liu QN, 2018 [40] & $\mathrm{RCT}$ & Unclear & Unclear & Yes & Yes & Yes & Yes & Unclear & Yes & Yes & 6 \\
\hline Li YF, 2015 [52] & $\mathrm{RCT}$ & Yes & Unclear & Yes & Yes & Yes & Yes & Unclear & Yes & Yes & 7 \\
\hline Ma GZ, 2019 [29] & $\mathrm{RCT}$ & Yes & Unclear & Yes & Yes & Yes & Yes & Unclear & Yes & Yes & 7 \\
\hline Peng Y, 2021 [15] & $\mathrm{RCT}$ & Yes & Unclear & Yes & Yes & Yes & Yes & Unclear & Yes & Yes & 7 \\
\hline $\mathrm{Tu}$ Y, 2018 [39] & $\mathrm{RCT}$ & Yes & Unclear & Yes & Yes & Yes & Yes & Unclear & Yes & Yes & 7 \\
\hline Wang L, 2019 [24] & $\mathrm{RCT}$ & Yes & Yes & Yes & No & Yes & Yes & Unclear & Yes & Yes & 7 \\
\hline Wang Q, 2018 [38] & RCT & Unclear & Unclear & Yes & Yes & Yes & Yes & Unclear & Yes & Yes & 6 \\
\hline Wu P, 2019 [28] & RCT & Unclear & Unclear & Yes & Yes & Yes & Yes & Unclear & Yes & Yes & 6 \\
\hline $\mathrm{Xu} \mathrm{YL,} 2020$ [10] & RCT & Yes & Unclear & Yes & Yes & Yes & Yes & Unclear & Yes & Yes & 7 \\
\hline Yao XQ, 2019 [27] & RCT & Yes & Unclear & Yes & Yes & Yes & Yes & Unclear & Yes & Yes & 7 \\
\hline You HQ, 2020 [18] & RCT & Yes & Unclear & Yes & Yes & Yes & Yes & Unclear & Yes & Yes & 7 \\
\hline Yu F, 2020 [17] & RCT & Yes & Unclear & Yes & Yes & Yes & Yes & Unclear & Yes & Yes & 7 \\
\hline Zhang JB, 2019 [26] & RCT & Unclear & Unclear & Yes & Yes & Yes & Yes & Unclear & Yes & Yes & 6 \\
\hline Zhang N, 2018 [37] & RCT & Yes & Unclear & Yes & Yes & Yes & Yes & Unclear & Yes & Yes & 7 \\
\hline Zhang RT, 2016 [47] & RCT & Unclear & Unclear & Unclear & Yes & Yes & Yes & Unclear & Yes & Yes & 5 \\
\hline Zhang XH, 2019 [25] & RCT & Yes & Unclear & Yes & Yes & Yes & Yes & Unclear & Yes & Yes & 7 \\
\hline Zhang Y, 2018 [36] & RCT & No & Unclear & Yes & Yes & Yes & Yes & Unclear & Yes & Yes & 6 \\
\hline Zhao FY, 2018 [35] & RCT & Yes & Unclear & Yes & Yes & Yes & Yes & Unclear & Yes & Yes & 7 \\
\hline Cheng LL, 2019 [32] & Non-RCT & Unclear & Unclear & Yes & Yes & Yes & Yes & Unclear & Yes & Yes & 6 \\
\hline Li YJ, 2019 [30] & Non-RCT & No & No & Yes & Yes & Yes & Yes & Unclear & Yes & Yes & 6 \\
\hline Ouyang HG, 2019 [9] & Non-RCT & No & No & Yes & Yes & Yes & Yes & Unclear & Yes & Yes & 6 \\
\hline Pan JY, 2015 [51] & Non-RCT & No & No & Yes & Yes & Yes & Yes & Unclear & Yes & Yes & 6 \\
\hline Qiao W, 2014 [53] & Non-RCT & No & No & Yes & Yes & Yes & Yes & Unclear & Yes & Yes & 6 \\
\hline Shi M, 2016 [45] & Non-RCT & No & No & Yes & Yes & Yes & Yes & Unclear & Yes & Yes & 6 \\
\hline Wang N, 2021 [14] & Non-RCT & No & No & Yes & Yes & Yes & Yes & Unclear & Yes & Yes & 6 \\
\hline Yang FH, 2017 [41] & Non-RCT & No & No & Yes & Yes & Yes & Yes & Unclear & Yes & Yes & 6 \\
\hline Zhang Y, 2016 [46] & Non-RCT & No & No & Yes & Yes & Yes & Yes & Unclear & Yes & Yes & 6 \\
\hline Zhong Y, 2020 [16] & Non-RCT & No & No & Yes & Yes & Yes & Yes & Unclear & Yes & Yes & 6 \\
\hline Chen GM, 2013 [54] & $\mathrm{CBA}$ & No & No & Yes & Yes & Yes & Yes & Unclear & Yes & Yes & 6 \\
\hline Gao YY, 2017 [44] & CBA & No & No & Yes & Yes & Yes & Yes & Unclear & Yes & Yes & 6 \\
\hline Hu GS, 2016 [49] & CBA & No & No & Yes & Yes & Yes & Yes & Unclear & Yes & Yes & 6 \\
\hline Li SF, 2010 [55] & CBA & No & No & Yes & Yes & Yes & Yes & Unclear & Yes & Yes & 6 \\
\hline Liu XM, 2016 [48] & CBA & No & No & Yes & Yes & Yes & Yes & Unclear & Yes & Yes & 6 \\
\hline Su QX, 2017 [42] & CBA & No & No & Yes & Yes & Yes & Yes & Unclear & Yes & Yes & 6 \\
\hline Wen XQ, 2015 [50] & CBA & No & No & Yes & Yes & No & Yes & Unclear & Yes & Yes & 5 \\
\hline
\end{tabular}

(1): random sequence generation; (2): allocation concealment; (3): baseline outcome measurements similar; (4): baseline characteristics similar; (5): incomplete outcome data; (6): knowledge of the allocated interventions adequately prevented during the study; (7): protection against contamination; (8): selective outcome reporting; (9): other risks of bias; CBA: controlled before-and-after trials; non-RCT: nonrandomized controlled trials; RCT: randomized controlled trials. 


\begin{tabular}{|c|c|c|c|}
\hline \multirow{2}{*}{\multicolumn{2}{|c|}{$\begin{array}{l}\text { Study } \\
\text { ID }\end{array}$}} & & \multirow{2}{*}{$\begin{array}{l}\% \\
\text { Weight }\end{array}$} \\
\hline & & SMD (95\% CI) & \\
\hline \multicolumn{4}{|l|}{ FPG } \\
\hline Bao JL, 2019 & $\rightarrow$ & $-1.63(-2.09,-1.18)$ & 4.08 \\
\hline Hao ML, 2019 & $\leftrightarrow$ & $-1.74(-2.23,-1.24)$ & 4.05 \\
\hline Li CF, 2017 & 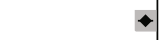 & $-0.49(-0.80,-0.17)$ & 4.18 \\
\hline Li YJ, 2019 & 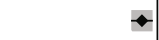 & $-0.64(-1.09,-0.19)$ & 4.08 \\
\hline Ouyang HG, 2019 & $\bullet$ & $-0.39(-0.47,-0.30)$ & 4.27 \\
\hline Pan JY, 2015 & - & $-0.68(-1.14,-0.21)$ & 4.07 \\
\hline Qiao W, 2014 & $\bullet$ & $-0.58(-0.86,-0.30)$ & 4.20 \\
\hline Tu Y, 2018 & $\bullet$ & $-0.87(-1.27,-0.48)$ & 4.13 \\
\hline Wang Q, 2018 & $\bullet$ & $-0.68(-1.06,-0.31)$ & 4.14 \\
\hline Wu P, 2019 & $\rightarrow$ & $-5.32(-6.12,-4.52)$ & 3.72 \\
\hline Yao XQ, 2019 & $\leftarrow$ & $-0.41(-0.62,-0.21)$ & 4.23 \\
\hline Zhang JB, 2019 & $\leftrightarrow$ & $-2.10(-2.54,-1.66)$ & 4.09 \\
\hline Zhang N, 2018 & 1 & $-4.82(-5.53,-4.11)$ & 3.83 \\
\hline Zhang RT, 2016 & \multirow[t]{2}{*}{$\bullet$} & $-0.79(-1.11,-0.47)$ & 4.18 \\
\hline Zhang XH, 2019 & & $-4.15(-4.65,-3.65)$ & 4.04 \\
\hline \multicolumn{2}{|r|}{$\leftarrow$} & $-2.19(-2.71,-1.66)$ & 4.02 \\
\hline Chen GM, 2013 & - & $-2.52(-2.77,-2.27)$ & 4.21 \\
\hline Su QX, 2017 & $\bullet$ & $-2.05(-2.25,-1.85)$ & 4.23 \\
\hline \multirow{2}{*}{\multicolumn{2}{|c|}{ 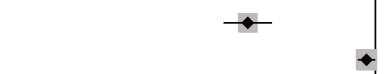 }} & $-5.25(-6.25,-4.25)$ & 3.48 \\
\hline \multirow{2}{*}{\multicolumn{2}{|c|}{$\leftrightarrow$}} & $-0.37(-0.73,-0.00)$ & 4.15 \\
\hline & & $-12.04(-14.18,-9.90)$ & 2.08 \\
\hline Xu YL, 2020 & $\bullet$ & $-0.66(-1.03,-0.29)$ & 4.15 \\
\hline \multicolumn{2}{|c|}{$\bullet$} & $-0.52(-0.96,-0.09)$ & 4.09 \\
\hline Chen YY, 2020 & $\bullet$ & $-0.85(-1.17,-0.54)$ & 4.18 \\
\hline Peng Y, 2021 & $\bullet$ & $-0.85(-1.26,-0.45)$ & 4.12 \\
\hline Subtotal $(\mathrm{I}-$ squared $=97.8 \%, \mathrm{p}=0.000)$ & $\diamond$ & $-1.85(-2.28,-1.42)$ & 100.00 \\
\hline \multicolumn{4}{|c|}{ NOTE: Weights are from random effects analysis } \\
\hline $\begin{array}{c}1 \\
-14.2\end{array}$ & 0 & & \\
\hline
\end{tabular}

FIGURE 2: Forest plot of the effect of health literacy intervention on FPG.

four studies $[10,21,37,55]$ showed that the excellent rate of knowledge HL was significantly boosted after the HL intervention $(P<0.05)$.

Taking these findings into consideration, we conclude that HL intervention has a significant promoting effect on general HL level in people with diabetes.

3.4. Meta-Analysis of Some Effect Indicators. A meta-analysis of the outcomes relating to glycemic indicators (FPG, 2hPG, and $\mathrm{HbAlc}$ ) and lipid indicators (TC, TG (triglycerides), LDL-C (low-density lipoprotein cholesterol), and HDL-C (high-density lipoprotein cholesterol)) was performed, and the results showed a high heterogeneity in the included studies. Because our analysis includes different types of study design, a subgroup analysis was performed of each indicator according to study design type. The results revealed little variation in heterogeneity, indicating that study design type was not the main cause of high heterogeneity.

\subsubsection{Glucose Metabolism}

(1) FPG. The effect of intervention on FPG level was reported in 25 studies $[9,10,15,16,18,21-23,25-28,30$, $31,33,35,37-39,42,43,47,51,53,54]$, with results showing that the intervention group is better than the control group in reducing FPG in patients $(\mathrm{SMD}=-1.85,95 \%$ CI $(-2.28$, -1.42), $P<0.05$; $Q$ statistic, $I^{2}=97.8 \%, P<0.1$ ) (Figure 2). The subgroup analysis also showed that different types of study design can effectively reduce FPG levels of patients in the intervention group (Figure S2).

(2) $2 h P G$. Nineteen studies $[10,15,16,18,21,22,25,26,28$, $31,33,35,37-39,42,47,53,54]$ presented data regarding the effect of HL intervention on $2 \mathrm{hPG}$ levels, showing a significant improvement in $2 \mathrm{hPG}$ in the intervention group compared with the control group $(\mathrm{SMD}=-2.18,95 \% \mathrm{CI}$ $(-2.68,-1.68), P<0.05 ; Q$ statistic, $\left.I^{2}=96.7 \%, P<0.1\right)$ (Figure 3). However, a subgroup analysis of two non-RCT studies $[16,53]$ showed that the HL interventions failed to significantly improve the $2 \mathrm{hPG}$ levels of patients, as shown in Figure S3.

(3) HbA1c. A total of 23 studies [9, 10, 15, 16, 21-28, 30, 31, $33,34,37-39,43,45,51,54]$ evaluated the impact of $\mathrm{HL}$ intervention on HbAlc levels. The HbAlc trend-changing graphs of two studies $[24,45]$ indicated that the improvement of HbAlc in the intervention group was better than 


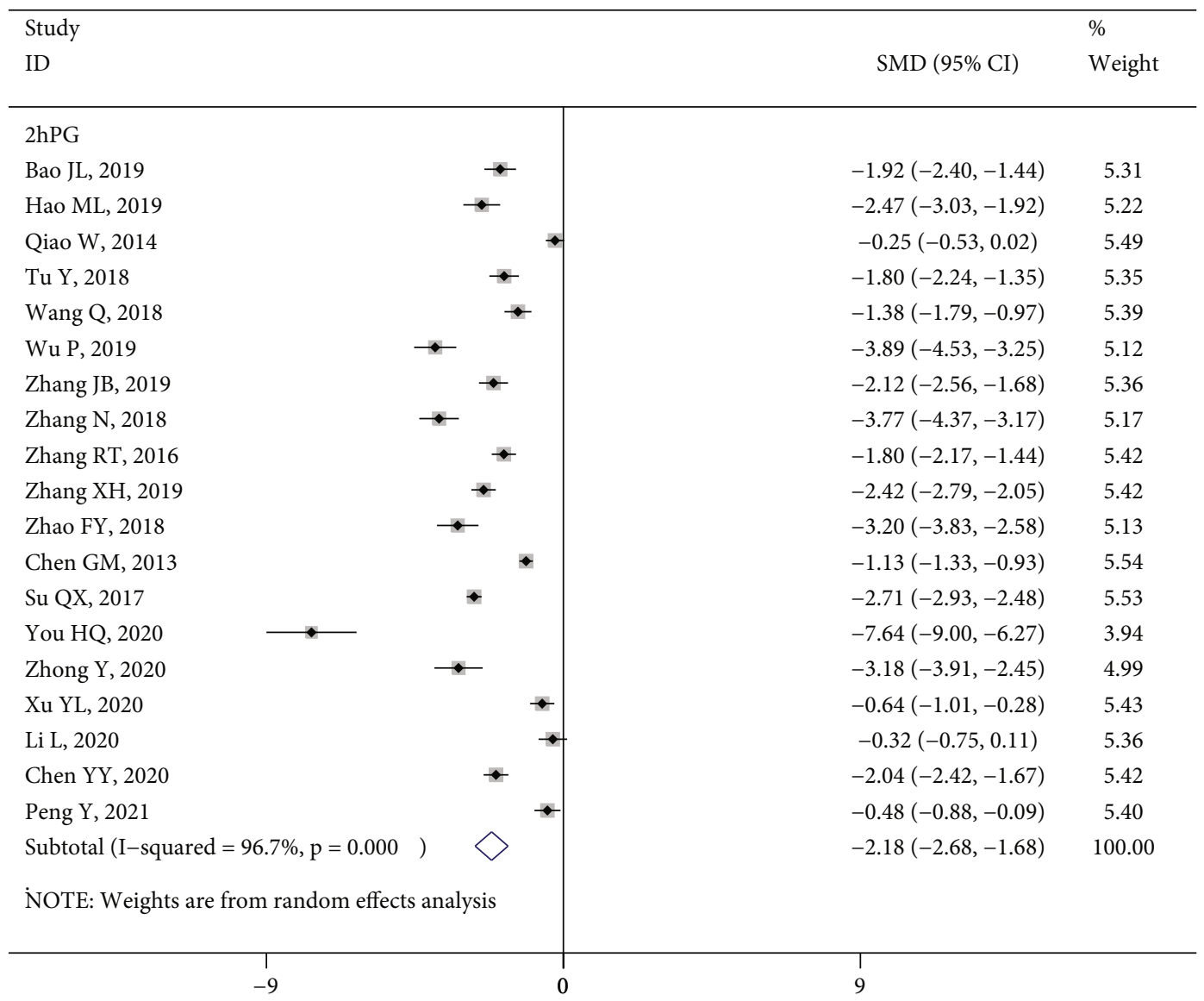

FIGURE 3: Forest plot of the effect of health literacy intervention on 2hPG.

that in the control group $(P<0.05)$. A meta-analysis of the relevant data in the other 21 studies showed heterogeneity $I^{2}=97.2 \%$, and the results of a random effect model revealed that the intervention group achieved better HbAlc control than the control group (WMD $=-1.21,95 \%$ CI $(-1.48$, $-0.94), P<0.05)$ (Figure 4$)$. The same results were also demonstrated in the subgroup analysis (Figure S4).

\subsubsection{Lipid Metabolism}

(1) TC. Seven studies $[9,10,15,27,38,39,43]$ included an analysis of the effect of HL intervention on TC levels. The results of the forest map showed that TC levels were significantly improved after $\mathrm{HL}$ intervention $(\mathrm{WMD}=-0.43$, 95\% CI $(-0.64,-0.23), P<0.05 ; Q$ statistic, $I^{2}=80 \%, P<$ 0.1 ) (Figure 5). The results are identical in the subgroup analyses (Figure S5).

(2) TG. Nine studies $[9,10,15,27,30,39,43,51,53]$ reported the impact of HL intervention on TG levels. The random effects model analysis showed that the improvement of TG in the HL intervention group was not statistically significant compared with the control group (WMD $=-0.34$, 95\% CI $(-0.73,0.05), P>0.05$; $Q$ statistic, $I^{2}=95.20 \%, P<$ 0.1 ) (Figure 6). However, a subgroup analysis of the RCT studies $[10,15,27,38,39,43]$ indicated that the HL inter- vention in the intervention group was effective in reducing the TG levels of patients compared with the control group (WMD $=-0.44,95 \% \mathrm{CI}(-0.82,-0.07), P<0.05)$, as shown in Figure S6.

(3) $L D L-C$. Six studies $[9,10,15,27,39,43]$ analyzed the impact of HL intervention on LDL-C levels $\left(I^{2}=86.8 \%\right.$, $P<0.1)$. The combined analysis and subgroup analysis performed by a random effect model all showed that there were no significant changes in LDL-C levels before and after HL intervention (WMD $=-0.20,95 \%$ CI $(-0.46,0.07)$, $P>0.05$ ) (Figure 7 and Figure S7).

(4) $H D L-C$. Six studies $[9,10,15,27,39,43]$ reported the impact of HL intervention on HDL-C $\left(I^{2}=94.7 \%, P<0.1\right)$. The parameters extracted from analysis using a random effect model indicated that HDL-C was not significantly improved by $\mathrm{HL}$ intervention (WMD $=-0.06,95 \% \mathrm{CI}$ $(-0.29,0.17), P>0.05)$ (Figure 8$)$. However, one non-RCT study [9] showed that the HDL-C level of the control group was better than that of the intervention group after the intervention (Figure S8).

3.5. Sensitivity Analysis. In order to ensure the stability of the meta-analysis conclusions, a sensitivity analysis was performed for each indicator using Stata software. After 


\begin{tabular}{|c|c|c|}
\hline \multirow{2}{*}{$\begin{array}{l}\text { Study } \\
\text { ID }\end{array}$} & \multirow{3}{*}{ WMD (95\% CI) } & \multirow{2}{*}{$\begin{array}{l}\% \\
\text { Weight }\end{array}$} \\
\hline & & \\
\hline \multicolumn{2}{|l|}{ HbAlc } & \\
\hline Bao JL, 2019 & $-0.08(-0.43,0.27)$ & 4.92 \\
\hline Hao ML, 2019 & $-0.92(-1.29,-0.55)$ & 4.87 \\
\hline Li CF, 2017 & $-1.35(-1.85,-0.85)$ & 4.54 \\
\hline Li YJ, 2019 & $-0.90(-1.39,-0.41)$ & 4.57 \\
\hline Ouyang HG, 2019 & $-1.30(-1.45,-1.15)$ & 5.27 \\
\hline Pan JY, 2015 & $-0.90(-1.36,-0.44)$ & 4.66 \\
\hline $\mathrm{Tu} \mathrm{Y}, 2018$ & $-1.81(-2.16,-1.46)$ & 4.93 \\
\hline Wang Q, 2018 & $-0.42(-0.64,-0.20)$ & 5.18 \\
\hline Wu P, 2019 & $-2.80(-3.45,-2.15)$ & 4.11 \\
\hline Yao XQ, 2019 & $-1.40(-1.80,-1.00)$ & 4.80 \\
\hline Zhang JB, 2019 & $-1.18(-1.67,-0.69)$ & 4.57 \\
\hline Zhang N, 2018 & $-2.60(-3.21,-1.99)$ & 4.22 \\
\hline Zhang XH, 2019 & $-0.71(-0.75,-0.67)$ & 5.35 \\
\hline Chen GM, 2013 & $-2.03(-2.15,-1.91)$ & 5.30 \\
\hline Cheng L, 2017 & $-0.66(-1.19,-0.13)$ & 4.45 \\
\hline Cao YF, 2020 & $-1.28(-1.45,-1.11)$ & 5.24 \\
\hline Zhong Y, 2020 & $-1.79(-2.43,-1.15)$ & 4.14 \\
\hline Xu YL, 2020 & $-0.44(-0.58,-0.30)$ & 5.28 \\
\hline Li L, 2020 & $-0.71(-1.39,-0.03)$ & 4.02 \\
\hline Chen YY, 2020 & $-1.71(-2.09,-1.33)$ & 4.86 \\
\hline Peng Y, 2021 & $-0.83(-1.26,-0.40)$ & 4.72 \\
\hline Subtotal $(\mathrm{I}-$ squared $=97.2 \%, \mathrm{p}=0.000)$ & $-1.21(-1.48,-0.94)$ & 100.00 \\
\hline \multicolumn{3}{|l|}{ NOTE: Weights are from random effects analysis } \\
\hline $\begin{array}{c}1 \\
-3.45\end{array}$ & 3.45 & \\
\hline
\end{tabular}

FIGURE 4: Forest plot of the effect of health literacy intervention on HbA1c.

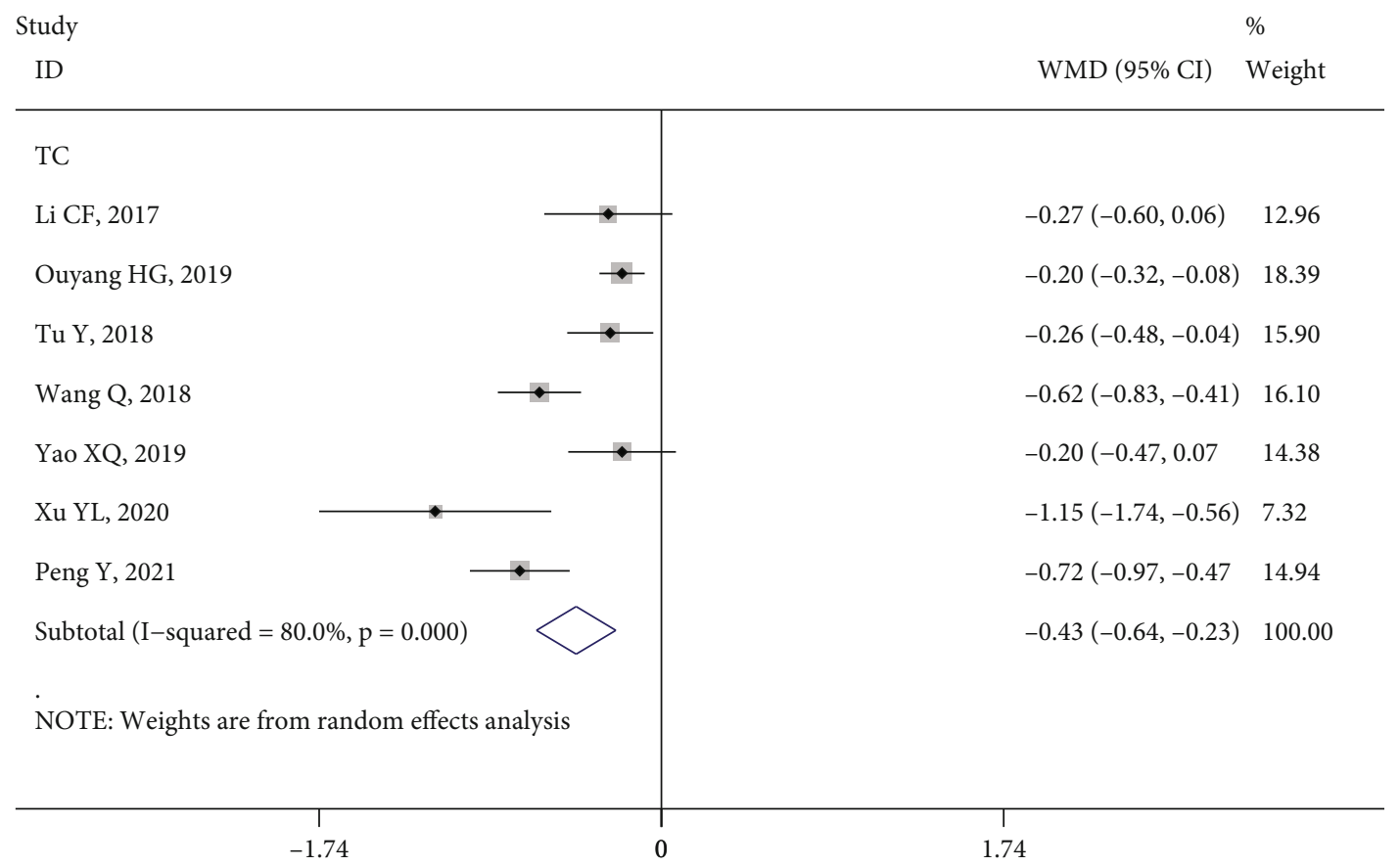

FIgURE 5: Forest plot of the effect of health literacy intervention on TC. 


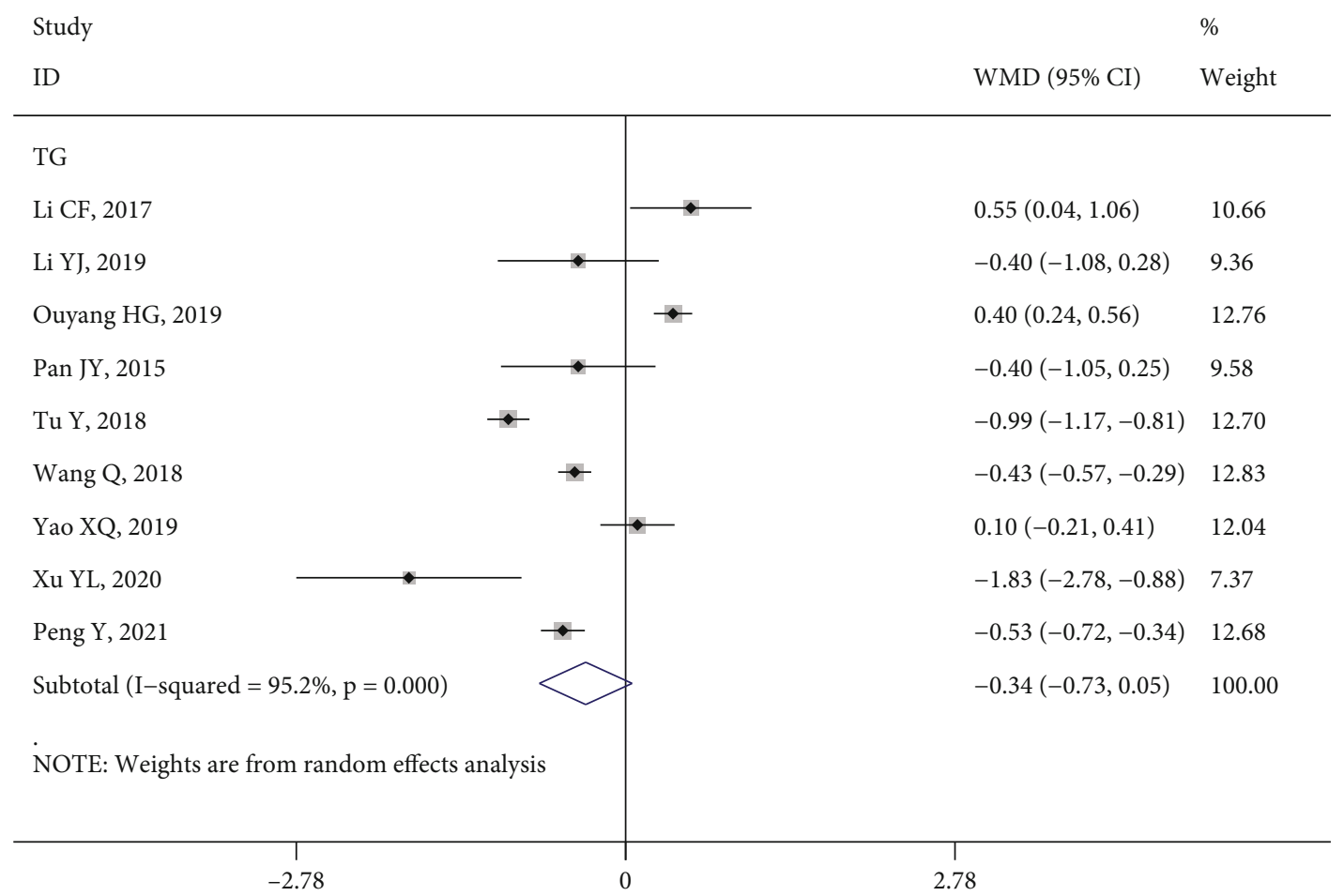

FIGURE 6: Forest plot of the effect of health literacy intervention on TG.

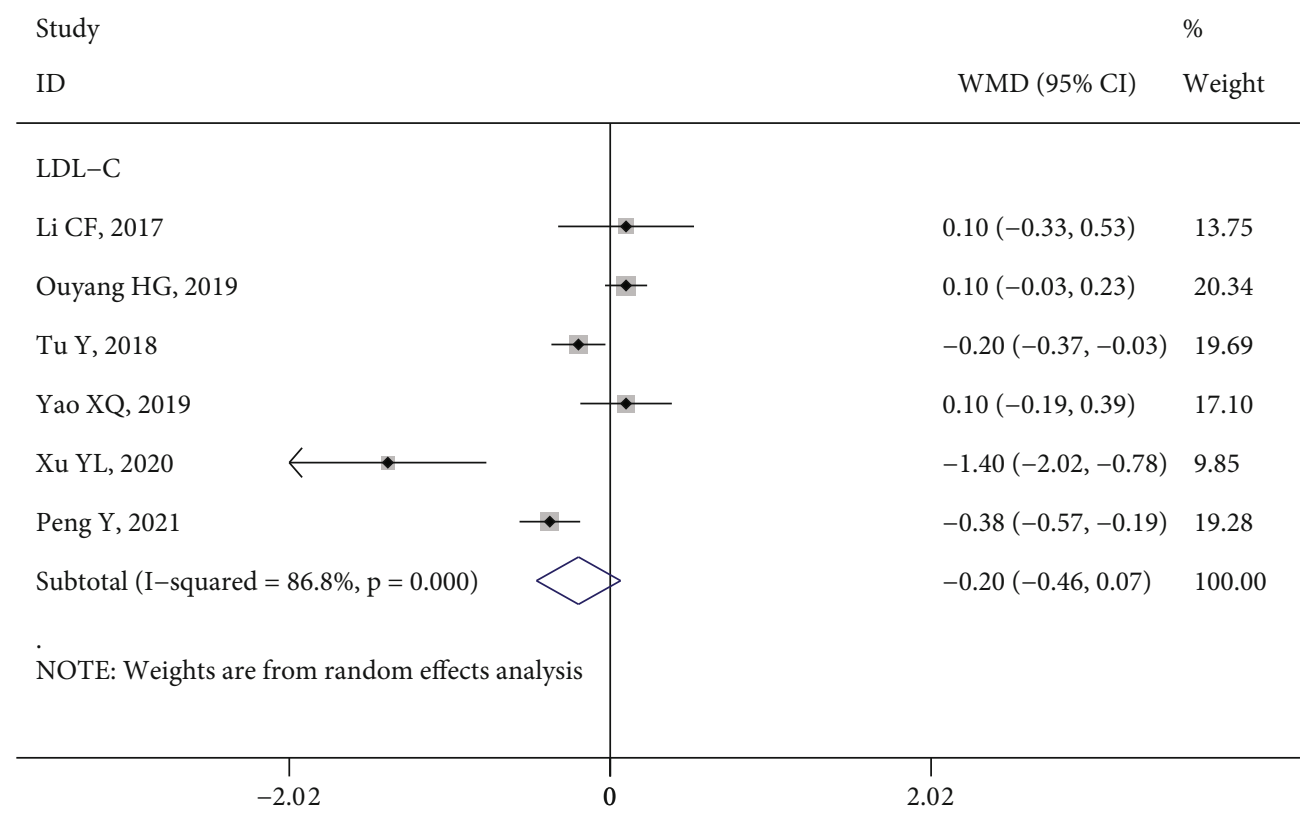

FIGURE 7: Forest plot of the effect of health literacy intervention on LDL-C.

removing any one study from each indicator, the results of the meta-analyses showed that the overall effect values of each indicator did not significantly skew the conclusions (Figure S9).

3.6. Publication Bias. Funnel plots were used for detecting publication bias. In this analysis, the funnel plots appeared to be asymmetrical, suggesting a certain publication bias
(Figure 9). The high heterogeneity and low-quality research methods included in the studies may be the reasons for any publication bias.

3.7. Grading of Quality of Evidence. Combined with relevant data, the certainty of evidence for each outcome was assessed using the GRADE approach, and a summary table was established using GRADEpro GDT software. Analysis 


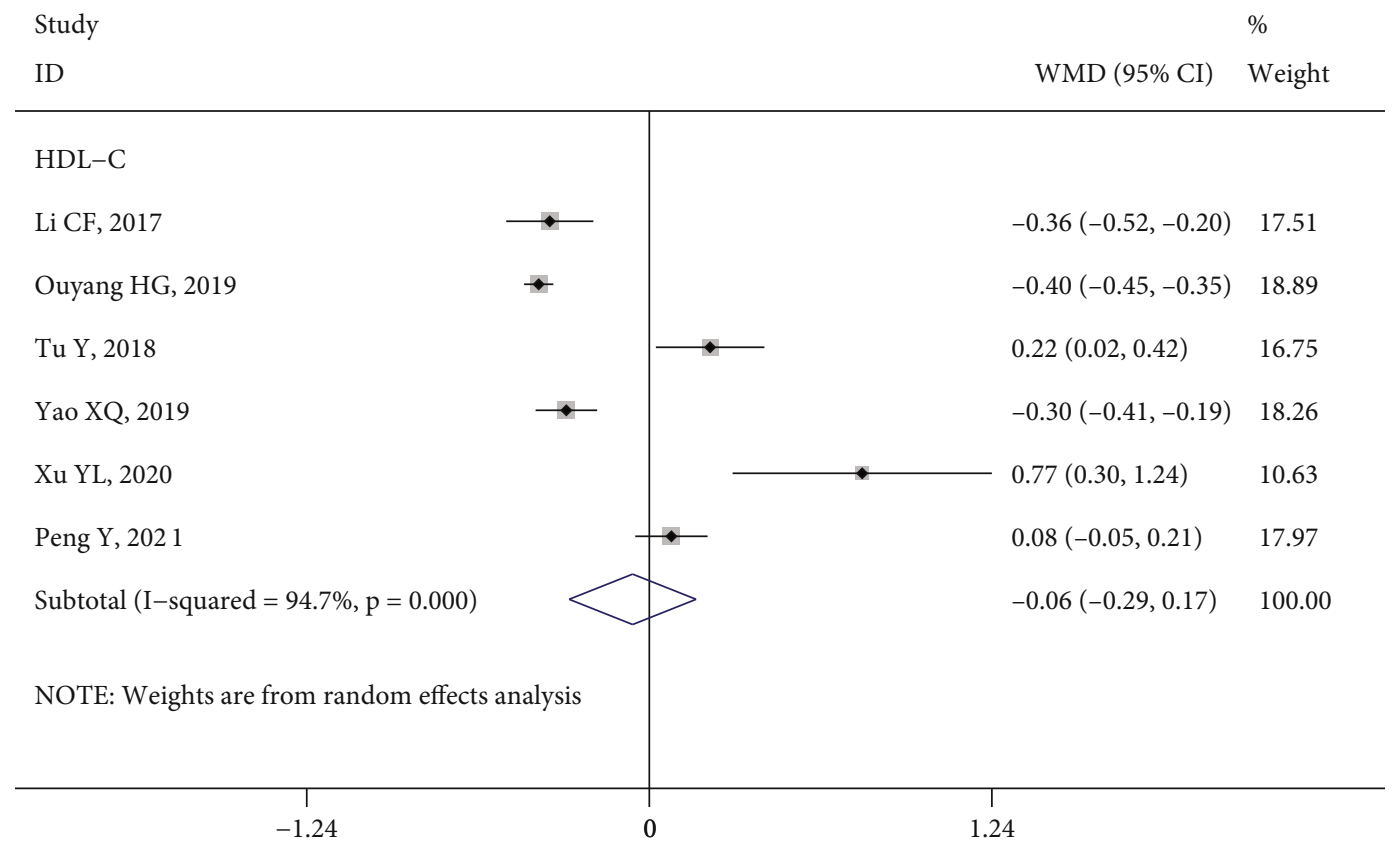

FIGURE 8: Forest plot of the effect of health literacy intervention on HDL-C.

indicated that the quality of evidence supporting the outcomes of the studies ranged from low to moderate due to inconsistency and publication bias (Table S4).

\section{Discussion}

To the best of our knowledge, this is the first SR of studies on $\mathrm{HL}$ intervention among people with diabetes in mainland China. Our findings indicate that HL intervention is effective in improving HL level and glycemic profile among people with diabetes, although improvements in lipid profile are less satisfactory.

4.1. Effectiveness of Intervention on $H L$. In general, it can be observed in our study that all patients who received the intervention showed a significant improvement in HL level. However, in terms of different dimensions, some patients only had an increase in HL knowledge level, and their calculation or application skills did not improve significantly [33, 43]. This may be related to the fact that interventions are mostly based on HE, which focuses excessively on knowledge indoctrination. It is a very complicated to achieve a qualitative change from accepting knowledge to using knowledge expertly [48]. As a result, it is possible that interventionists neglected to strengthen and validate patients' calculations or application skills during the intervention. Second, it is also possible that the duration of the intervention was too short to lead to such an outcome. Behavior change is a difficult process that requires continuous intervention and reinforcement of multiple factors over time, and the desired change may not be achieved through short-term training [56]. This phenomenon also suggests that future HL interventions should focus not only on the output of knowledge but also on the education and exercises in skills and application.
4.2. Effectiveness of Intervention on Glycolipid Metabolism. Previous studies have confirmed that the improvements in self-management are conducive to blood sugar control among people with diabetes [57]. Compared with conventional care, HL intervention can help patients distinguish their different diabetes care needs, thereby improving the relevance of care and their level of self-management. This is a positive factor that provides theoretical support for the self-management of people with diabetes. The results of our study also confirm this point: the level of selfmanagement of patients was strengthened after HL intervention such that the control of FPG, 2hPG, and HbAlc was improved.

Disturbed glucose metabolism in diabetes predisposes to disturbed lipid metabolism, which is itself an independent risk factor for various chronic diabetes complications [58]. Consequently, previous studies have analyzed the effects of HL intervention on lipids in patients with diabetes. Some studies have demonstrated that HL interventions are effective in controlling the lipid profile of patients [15], while others have suggested that HL interventions were not beneficial for lipid control [43]. The structure of the diet, the mode of exercise, and the timing of the intervention may all contribute to this conflict. Diet and exercise are two healthy means of ameliorating blood lipid levels, but both approaches require a considerable amount of time to show an effect, with significant effects not necessarily occurring over a period of 3 or 6 months [59]. In the present study, HL intervention was not effective in controlling the lipid profile of people with diabetes.

4.3. Sources of Heterogeneity. In this meta-analysis, heterogeneity between studies was high, and neither the subgroup analysis of study design type nor the sensitivity analysis was able to identify sources of heterogeneity. Therefore, we 
Funnel plot with pseudo 95\% confidence limits

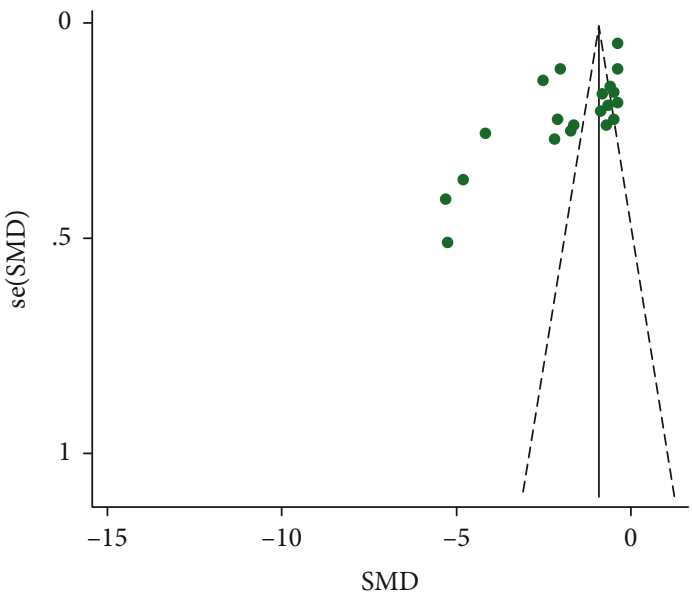

(a)

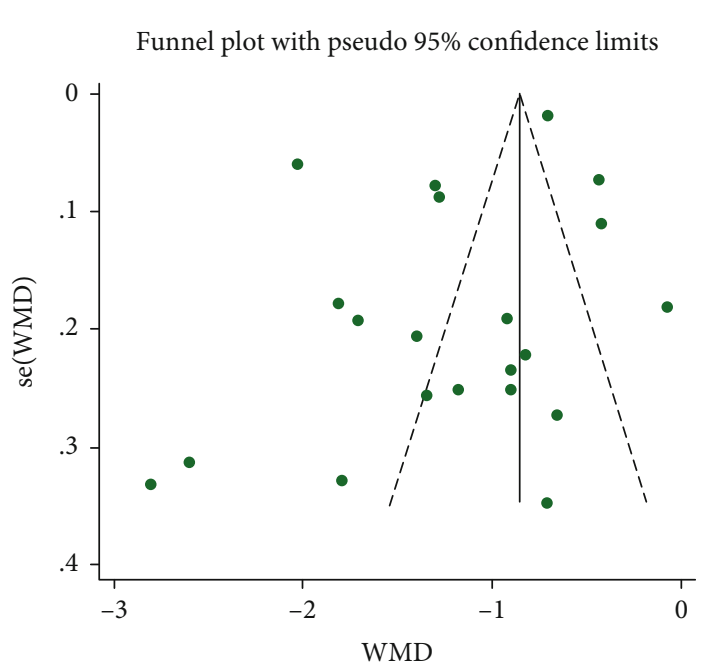

(c)

Funnel plot with pseudo 95\% confidence limits

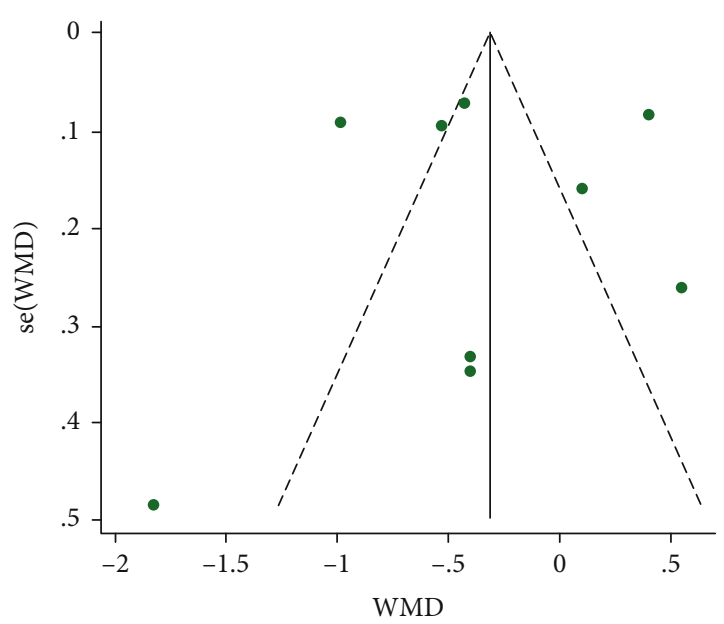

(e)
Funnel plot with pseudo 95\% confidence limits

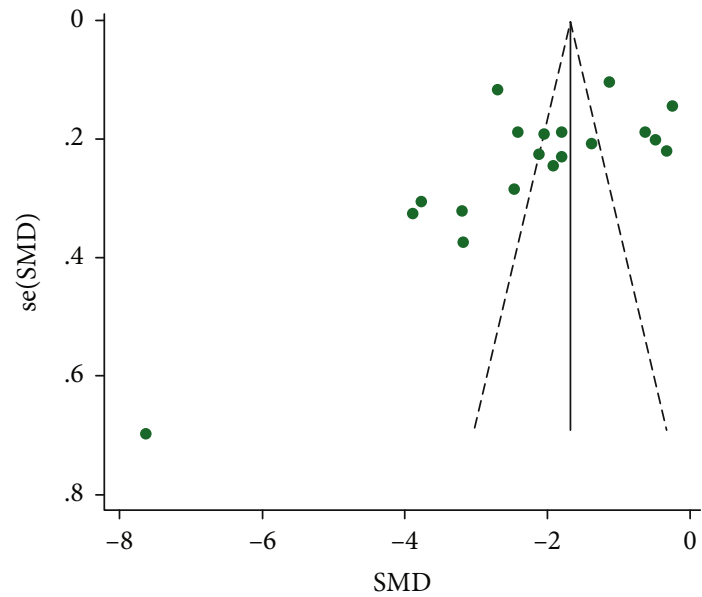

(b)

Funnel plot with pseudo 95\% confidence limits

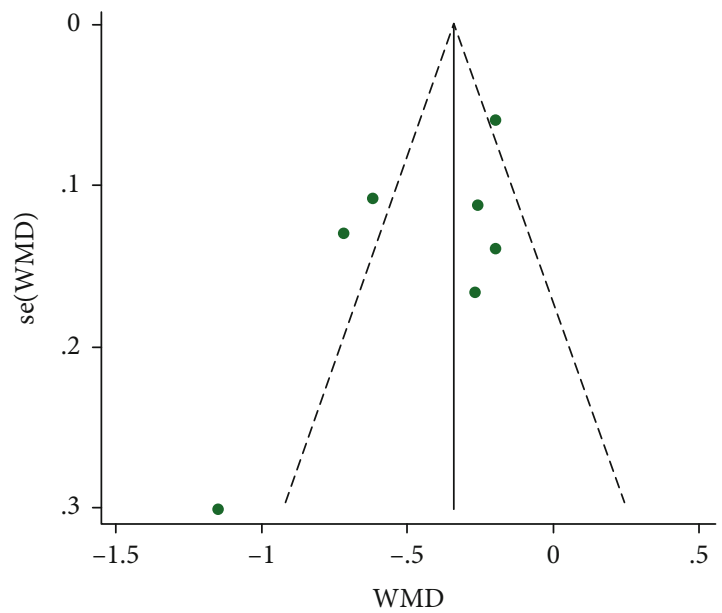

(d)

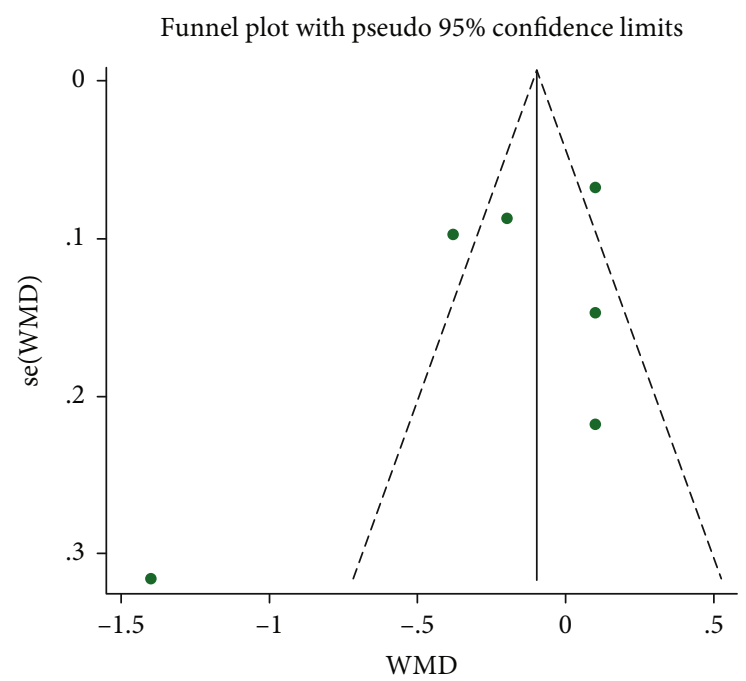

(f)

FIgURE 9: Continued. 


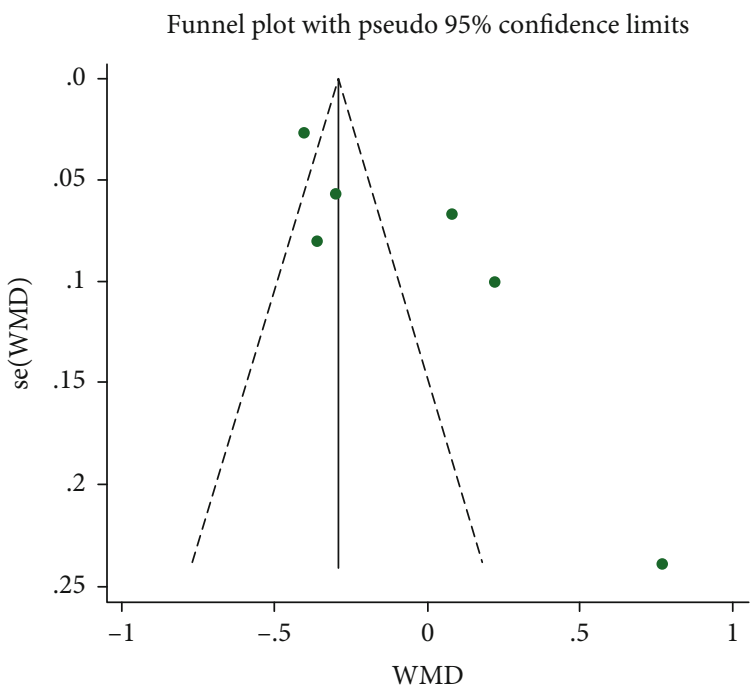

(g)

Figure 9: Funnel plots of intervention effect indexes ((a) FPG; (b) 2hPG; (c) HbA1c; (d) TC; (e) TG; (f) LDL-C; (g) HDL-C).

further considered clinical aspects of heterogeneity. First, the interventions included differed between studies, with some focusing on dietary education while others adopted a teach-back approach to education, for example. Second, baseline HL levels differed among participants, which may potentially affect the effectiveness of the intervention. Third, the different methods of measuring indicator data could have an impact on outcomes. Fourth, the overall low assessment of methodological quality in the included studies may also lead to a high level of heterogeneity. Moreover, several studies showed that women and ethnic minorities were often underrepresented in clinical trials testing cardiovascular drugs, which may limit the generalizability of trial results to the entire population $[60,61]$. This phenomenon reminds that the inequalities in enrollment of women and ethnic minorities in this study could lead to heterogeneity. As the studies included in this SR and meta-analysis did not provide data on the outcomes between men and women and ethnicity, we were unable to conduct further research on them. It is recommended that future HL intervention studies need to enhance the representativeness of clinical trials according to race and sex.

4.4. Innovations and Limitations. This study has a number of innovations. First, this study is the first to use an evidencebased medicine approach to systematically and comprehensively analyze multiple types of intervention for diabetes HL in mainland China and to quantitatively evaluate the improvement in glucose and lipid metabolism through meta-analysis to achieve a comprehensive evaluation of patient HL levels. Second, the majority of available studies adopted improvement in patient blood glucose as the evaluation index, with a lack of consideration for blood lipid evaluation. In addition to the analysis of blood glucose, this SR also conducted a combined analysis of changes in blood lipids after the intervention and more comprehensively evaluated the impact of HL intervention on biochemical indicators. Third, this study includes a comprehensive analysis and discussion of various HL assessment tools and elaborates on the improvement in patient HL after HL intervention.

The limitations of this study include the lack of a registration number in a SR registration platform (e.g., PROSPERO) for prereview, which may affect the transparency of the study, while the analysis of heterogeneity is not sufficiently comprehensive. Subgroup analysis was conducted only according to the type of study design, and subsequent analyses in the future could consider this from other perspectives, such as intervention method and intervention time.

\section{Conclusion}

The HL intervention enhanced the total health literacy level of people with diabetes and effectively improved glucose metabolism and TC levels, but did not significantly improve TG, LDL-C, or HDL-C. Subsequent study designs should include more scientific and rigorous high-quality intervention plans, including extending the intervention and follow-up time, considering the impact on patient behavior and skills, and clearing the long-term effect of HL. In addition, a set of diabetes HL evaluation index systems applicable to the population in mainland China should be established as soon as possible, so as to provide evaluation criteria and implementation references for the formulation of HL interventions for people with diabetes.

\section{Conflicts of Interest}

No potential conflicts of interest relevant to this article were reported.

\section{Authors' Contributions}

Kui Jiang contributed to the conception and design of this study and controlled the quality of the manuscript. Yangli Chen performed the literature search, literature screening, 
and data extraction and wrote the manuscript. Xue Ran participated in literature screening and data extraction. Yalan Chen supervised the quality of data and the manuscript. Kui Jiang is the guarantor of this work and, as such, had full access to all the data in the study and takes responsibility of the integrity of the data and the accuracy of the data analysis.

\section{Acknowledgments}

This work was supported by Postgraduate Research \& Practice Innovation Program of Jiangsu Province (KYCX21_ 3105).

\section{Supplementary Materials}

Table S1: PRISMA checklists. Table S2: search strategy. Table S3: contents of health literacy assessment tools. Table S4: summary of finding table. Figure S1: usage distribution of health literacy assessment tools. Figure S2: forest plot of FPG by subgroup analysis. Figure S3: forest plot of $2 \mathrm{hPG}$ by subgroup analysis. Figure S4: forest plot of $\mathrm{HbA1c}$ by subgroup analysis. Figure S5: forest plot of TC by subgroup analysis. Figure S6: forest plot of TG by subgroup analysis. Figure S7: forest plot of LDL-C by subgroup analysis. Figure S8: forest plot of HDL-C by subgroup analysis. Figure S9: sensitivity analysis of intervention effect indexes (A: FPG; B: 2hPG; C: HbAlc; D: TC; E: TG; F: LDL-C; G: HDL-C). (Supplementary Materials)

\section{References}

[1] International Diabetes Federation, IDF Diabetes Atlas, International Diabetes Federation, Brussels, Belgium, 9th edition, 2019.

[2] L. F. Wu, Y. L. Li, Q. H. Zhou, and Q. Q. Lou, "Effects of diabetes education in mainland China: a meta-analysis," Journal of Nursing Science, vol. 25, no. 17, pp. 79-83, 2010.

[3] C. Y. Li, X. S. Zhao, M. Kim, J. Y. Quan, C. Y. Li, and C. F. Li, "Impact of disease-related health literacy on the control of blood glucose in community patients with diabetes mellitus," Chinese Journal of Nursing, vol. 47, no. 1, pp. 69-71, 2012.

[4] X. N. Liu, R. Li, Q. H. Xia, H. Fang, and W. H. Xu, "Research progress on health literacy of patients with type 2 diabetes," Journal of Environmental \& Occupational Medicine, vol. 33, no. 9, pp. 900-905, 2016.

[5] J. G. Souza, D. Apolinario, R. M. Magaldi, A. L. Busse, F. Campora, and W. Jacob-Filho, "Functional health literacy and glycaemic control in older adults with type 2 diabetes: a cross-sectional study," BMJ Open, vol. 4, no. 2, article e004180, 2014.

[6] C. Y. Osborn, K. Cavanaugh, K. A. Wallston, and R. L. Rothman, "Self-efficacy links health literacy and numeracy to glycemic control," Journal of Health Communication, vol. 15, supplement 2, pp. 146-158, 2010.

[7] M. T. Kim, K. B. Kim, B. Huh et al., "The effect of a community-based self-help intervention: Korean Americans with type 2 diabetes," American Journal of Preventive Medicine, vol. 49, no. 5, pp. 726-737, 2015.

[8] S. Chamany, E. A. Walker, C. B. Schechter et al., “Telephone intervention to improve diabetes control: a randomized trial in the New York City A1c Registry," American Journal of Preventive Medicine, vol. 49, no. 6, pp. 832-841, 2015.

[9] H. G. Ouyang and Y. L. Liang, "Intervention study on community type 2 diabetes mellitus patients' health literacy by individualized health education model based on 'three division co-management'," Laboratory Medicine and Clinic, vol. 16, no. 2, pp. 204-207, 2019.

[10] Y. L. Xu, M. H. Liu, L. P. Dong, J. W. Zhang, and F. Ma, "Impact of community-hospital-family intervention on blood glucose level and self-management in diabetic patients," International Journal of Geriatrics, vol. 41, no. 2, pp. 114-117, 2020.

[11] M. J. Page, J. E. McKenzie, P. M. Bossuyt et al., "The PRISMA 2020 statement: an updated guideline for reporting systematic reviews," BMJ, vol. 372, 2021.

[12] Cochrane Effective Practice and Organisation of Care (EPOC), "Suggested risk of bias criteria for EPOC reviews," EPOC Resources for review authors, 2017, http://www.epoc .cochrane.org/resources/epoc-resources-review-authors.

[13] H. J. Schünemann, J. P. Higgins, G. E. Vist et al., "Completing 'summary of findings' tables and grading the certainty of the evidence," in Cochrane Handbook for Systematic Reviews of Interventions, pp. 375-402, John Wiley \& Sons, Chichester (UK), 2019.

[14] N. Wang, X. P. Bai, and S. X. Li, "Effects of teach-back method combined with video education on the knowledge, belief, behavior and health literacy of elderly diabetic patients," Harbin Medical Journal, vol. 41, no. 1, pp. 110-111, 2021.

[15] Y. Peng, Y. Lin, H. Zhou, F. Tian, Y. Y. Pan, and K. X. Li, "Studying on the influence of Internet-based health education on the health literacy and self-management ability of patients with type 2 diabetes mellitus in communities," Chinese Health Service Management, vol. 38, no. 3, pp. 237-240, 2021.

[16] Y. Zhong, "The effect of health promotion management mode intervention on the health literacy of the elderly with diabetes in community," Journal of Frontiers of Medicine, vol. 10, no. 9, pp. 243-244, 2020.

[17] F. Yu, "Effects of insulin interview tool on health literacy and prognosis of elderly diabetic patients," Today Nurse, vol. 27, no. 7, pp. 159-161, 2020.

[18] H. Q. You, "Study on intervention of structured health education model on health literacy of elderly cadres with type 2 diabetes mellitus," Diabetes World, vol. 17, no. 7, p. 4, 2020.

[19] Q. H. Liu, "Effects of five-full nursing modes on health management of elderly patients newly diagnosed with type 2 diabetes mellitus," Our Health, vol. 8, pp. 256-257, 2020.

[20] L. Li, X. H. Tang, L. Lai, and Q. Yang, "The effect of teach-back method combined with family involved on health literacy and quality of life in elderly diabetic patients," Anhui Medical Journal, vol. 41, no. 12, pp. 1471-1475, 2020.

[21] L. Li, L. Lai, X. H. Tang, Q. Yang, and L. Zhao, "The effects of teach-back method combined with telephone follow-up on health literacy and blood glucose control in rural diabetic patients," Journal of North Sichuan Medical College, vol. 35, no. 2, pp. 336-339, 2020.

[22] Y. Y. Chen and X. Huang, "Application of teach-back method in elderly patients with type 2 diabetes," Health Vocational Education, vol. 38, no. 16, pp. 136-138, 2020.

[23] Y. F. Cao and F. Li, "Research on the effect of WeChat health education on healthy lifestyle of elderly diabetic," Chinese Journal of Health Education, vol. 36, no. 10, pp. 954-957, 2020. 
[24] L. Wang, H. Fang, Q. H. Xia et al., "Health literacy and exercise-focused interventions on clinical measurements in Chinese diabetes patients: a cluster randomized controlled trial," EClinicalMedicine, vol. 17, article 100211, 2019.

[25] X. H. Zhang, "The effect of continuous health education combined with exercise intervention on discharged patients with type 2 diabetes," Medical Journal of Chinese People's Health, vol. 31, no. 16, pp. 156-158, 2019.

[26] J. B. Zhang, "Discussion on the application of selfmanagement group in community patients with diabetes," Electronic Journal of Practical Clinical Nursing Science, vol. 4, no. 38, pp. 177-178, 2019.

[27] X. Q. Yao, Y. P. Liu, L. Wang, P. Shuai, H. Yang, and Z. Tang, "Study on intervention of structured health education model on health literacy of elderly cadres with type 2 diabetes mellitus," Journal of Modern Medicine and Health, vol. 35, no. 24, pp. 3746-3748+3754, 2019.

[28] P. Wu and S. M. Zhang, "Observation on the effect of microclass health education on improving the health literacy of diabetic patients," Medical World, vol. 17, p. 171, 2019.

[29] G. Z. Ma, B. R. Mo, H. L. Deng et al., "Five-full nursing modes in health management of elderly patients newly diagnosed with type 2 diabetes mellitus," International Medicine \& Health Guidance News, vol. 25, no. 12, pp. 1875-1878, 2019.

[30] Y. J. Li, "Clinical observation of whole-course dietary management intervention on type 2 diabetes patients," China's Naturopathy, vol. 27, no. 21, pp. 65-67, 2019.

[31] M. L. Hao, M. S. Cui, and M. Liu, "Application of health education based on teach-back theory in patients with type 2 diabetes," Journal of Qilu Nursing, vol. 25, no. 17, pp. 90-93, 2019.

[32] L. L. Cheng, "Observation on the effect of strengthening selfefficacy management in community patients with type 2 diabetes," Journal of Frontiers of Medicine, vol. 9, no. 5, pp. 243-244, 2019.

[33] J. L. Bao, B. L. Sun, M. Y. Li, L. Li, J. L. Zhou, and W. D. Huang, "Application of teach-back method in health education for newly diagnosed elderly patients with type 2 diabetes mellitus," Journal of Changchun University of Chinese Medicine, vol. 35, no. 3, pp. 556-559, 2019.

[34] L. Cheng, J. W. Sit, K. C. Choi et al., "Effectiveness of a patientcentred, empowerment-based intervention programme among patients with poorly controlled type 2 diabetes: a randomised controlled trial," International Journal of Nursing Studies, vol. 79, pp. 43-51, 2018.

[35] F. Y. Zhao, "The impact of empowerment education on healthrelated literacy and blood sugar control of diabetic patients," Today Nurse, vol. 25, no. 4, pp. 25-27, 2018.

[36] Y. Zhang, "The effect of family doctors' comprehensive health literacy management on type 2 diabetes patients," Psychological Doctor, vol. 24, no. 25, pp. 344-345, 2018.

[37] N. Zhang, "Observation on the effect of micro-class health education on improving the health literacy of diabetic patients," Modern Practical Medicine, vol. 30, no. 4, pp. 548549, 2018.

[38] Q. Wang, "Effects of teach-back health education on health literacy and glucose and lipid metabolism of type 2 diabetic patients with poor glycemic control," Nursing Practice Research, vol. 15, no. 17, pp. 22-24, 2018.

[39] Y. Tu, L. Cheng, L. P. Ou, and Y. M. Chen, "The application of health management in elderly patients with type 2 diabetes mellitus under the integrated intervention of family doctor,"
International Medicine \& Health Guidance News, vol. 24, no. 17, pp. 2593-2596, 2018.

[40] Q. N. Liu, W. L. Ye, X. H. Chen, S. X. Li, A. W. Chen, and $\mathrm{X}$. Huang, "Research on improving the utilization of healthy literacy of patients with diabetes and its research on the utilization of community health service," China Medicine and Pharmacy, vol. 8, no. 20, pp. 219-221, 2018.

[41] F. H. Yang, W. Shen, X. P. Zhao, R. Liu, D. R. Peng, and G. Y. $\mathrm{Wu}$, "Effect of family doctors diabetes health literacy management on improving the level of patients health," Chinese Primary Health Care, vol. 31, no. 8, pp. 36-38, 2017.

[42] Q. X. Su, C. F. Zhang, N. Huang, X. W. Liang, and W. Wu, "The effect of comprehensive intervention on the health literacy of the residents on the patients with diabetes mellitus," Journal of Preventive Medicine Information, vol. 33, no. 9, pp. 869-872, 2017.

[43] C. F. Li, "The effect of self-management intervention on the health literacy of middle-aged and elderly diabetic patients in the community," Chinese Journal of Gerontology, vol. 37, no. 11, pp. 2824-2826, 2017.

[44] Y. Y. Gao, A. H. Yue, B. Zhang et al., "Evaluation on the effect of intervention with the chronic disease management team for type 2 diabetes patients in the community," China Health Care \& Nutrition, vol. 27, no. 24, p. 58, 2017.

[45] M. Shi, M. Y. Xu, Z. L. Liu et al., "Effectiveness of family involvement in newly diagnosed type 2 diabetes patients: a follow-up study," Patient Education and Counseling, vol. 99, no. 5, pp. 776-782, 2016.

[46] Y. Zhang, H. Z. Wang, and Y. L. Gao, "Application of microclasses in health literacy training for elderly diabetic patients," Guangdong Medical Journal, vol. 37, no. 16, pp. 2513-2515, 2016.

[47] R. T. Zhang and H. P. Zhou, "Evaluation of effect of mental intervention on health literacy of patients with precommunity diabetes," China Health Industry, vol. 13, no. 22, pp. 196-198, 2016.

[48] X. M. Liu, Y. F. Zhang, J. X. Sun, and D. X. Wang, "Influence of community health education on disease control of diabetic patients," Journal of Medical Theory and Practice, vol. 29, no. 1, pp. 123-125, 2016.

[49] G. S. Hu, "Analysis of the utilization of community health services of diabetic patients in the community after health literacy intervention," Guangzhou Medical Journal, vol. 47, no. 3, pp. 96-98, 2016.

[50] X. Q. Wen, Z. Z. Han, J. H. Liu et al., "Influence of the utilization of community health service on improving the health literacy of patients with diabetes mellitus," Chinese General Practice, vol. 18, no. 33, pp. 4025-4033, 2015.

[51] J. Y. Pan, J. M. Guo, R. S. Tan, X. Q. Xiong, and Z. B. Liang, "Effects of whole-course dietary management intervention on patients with type 2 diabetes mellitus," Chinese Journal of Public Health Management, vol. 31, no. 1, pp. 86-88, 2015.

[52] Y. F. Li, Y. B. Liu, and L. Liu, "Evaluation of education effects of teach-back method on senior citizens' diabetes related health literacy in nursing homes," Chinese Journal of Nursing, vol. 50, no. 11, pp. 1383-1387, 2015.

[53] W. Qiao, M. C. Ni, J. Luan, and Y. Bao, "Influence of village clinic construction on control effect in rural patients with diabetes," Occupation and Health, vol. 30, no. 18, pp. 2636-2638, 2014. 
[54] G. M. Chen, "Observation on the community nursing intervention effect of diabetes B," China Medicine and Pharmacy, vol. 3, no. 17, pp. 163-164, 2013.

[55] S. F. Li, "Impact of a nurse-led clinic on health literacy of diabetic patients," Journal of Nursing Science, vol. 25, no. 13, pp. 43-44, 2010.

[56] J. J. Hu, W. H. Zhang, W. L. Luo, Y. F. Sui, and J. J. Wang, "Research progress of behavior change theory applied to nursing intervention for chronic disease patients," Chinese Evidence-Based Nursing, vol. 5, no. 9, pp. 804-806, 2019.

[57] C. A. Chrvala, D. Sherr, and R. D. Lipman, "Diabetes selfmanagement education for adults with type 2 diabetes mellitus: a systematic review of the effect on glycemic control," Patient Education and Counseling, vol. 99, no. 6, pp. 926943, 2016.

[58] T. T. Yu, X. L. Yu, L. M. Zeng, X. Zhou, and R. H. Zhao, "Baduanjin for diabetes: a systematic review," Chinese Journal of Evidence-Based Medicine, vol. 14, no. 3, pp. 341-348, 2014.

[59] E. R. Vieira, F. A. D. C. Cavalcanti, F. Civitella et al., "Effects of exercise and diet on body composition and physical function in older Hispanics with type 2 diabetes," International Journal of Environmental Research and Public Health, vol. 18, no. 15, p. 8019, 2021.

[60] F. Fogacci, D. Gori, and A. F. G. Cicero, "Representativity of women and racial/ethnic minorities in randomized clinical trials on bempedoic acid: positive efforts and lacking data," European Journal of Internal Medicine, vol. S0953-6205, no. 21, 2021.

[61] F. Fogacci, C. Borghi, A. Di Micoli, D. Degli Esposti, and A. F. G. Cicero, "Inequalities in enrollment of women and racial minorities in trials testing uric acid lowering drugs," Nutrition, Metabolism, and Cardiovascular Diseases, vol. 31, no. 12, pp. 3305-3313, 2021. 